\title{
Rollman and Brent: Phonotype
}

\author{
Thomas N. Robinson, $M D, M P H^{7}$, Byron Reeves, $P h D^{2}$, and Nilam Ram, $P h D^{3}$ \\ 'Departments of Pediatrics and Medicine, Stanford Solutions Science Lab, Stanford University, Stanford, CA, USA; ${ }^{2}$ Department of Communication, \\ Stanford University, Stanford, CA, USA; ${ }^{3}$ Departments of Human Development and Family Studies and Psychology, Pennsylvania State University, \\ State College, PA, USA.
}

$\mathrm{J}$ Gen Intern Med 35(8):2479

DOI: $10.1007 / \mathrm{s} 11606-020-05798-y$

(C) Society of General Internal Medicine 2020

$\mathrm{D}$ ear Editor:

We agree with the authors' enthusiasm for using data collected unobtrusively from smartphones to detect patterns associated with states of health and disease, improve screening and diagnosis, and fulfill the promise of precision health with truly personalized, just-in-time adaptive interventions. ${ }^{1}$ Many useful diagnostic signals embedded in individuals' digital behavior will come through interaction with personal screens. Indeed, evidence of the patterns mentioned in the article (e.g., word choices, social media content, velocity of switching and input) do appear on those screens. The term "phonotype," however, emphasizes the device rather than the screens, through which individuals actually interface with the digital world. We propose the term "screenotype." Screenotypes are derived from the "screenome," the unique individual record of experiences that constitute physical, psychological, and social life on digital devices with screens - the study of which is "screenomics." We prefer the prefix screen-because it is the screen, not the phone, that serves as the primary expression of individuals' digital exposures and behaviors.

Through our research collecting screenomes from hundreds of individuals, we have identified a growing number of screenotypes that capture potentially important both between-person differences (e.g., diversity of applications used or visual interfaces engaged) and within-person dynamics (e.g., diurnal patterns in information flow) that will potentially support personalized and time- and context-sensitive micro-interventions. Notably, individuals' digital lives are characterized by speedy transitions between screens on many different devices (e.g., between smartphone and laptop, and potentially include transitions to screens on tablets, cable systems, and even cars). People experience digital life as threads that they actively construct and that weave seamlessly across multiple screens and often across radically different contents and possibilities that emerge on all of them. ${ }^{2}$ Fully subscribing to the analogy to genomics and other omics, we suggest maintaining accuracy and infusing poetry in the emerging science through generalization beyond the phonotype, which may evoke imagery related to an oldschool record player, to the screenotype, screenome, and screenomics.

Sincerely,

Thomas N. Robinson, MD, MPH

Departments of Pediatrics and Medicine, Stanford University

Byron Reeves, $\mathrm{PhD}$

Department of Communication, Stanford University

Nilam Ram, PhD

Departments of Human Development and Family Studies and Psychology, Pennsylvania State University

Corresponding Author: Thomas N. Robinson, MD, MPH; Departments of Pediatrics and Medicine, Stanford Solutions Science Lab, Stanford University, Stanford, CA, USA (e-mail: tom. robinson@stanford.edu).

\section{Compliance with ethical standards:}

Conflict of Interest: The authors declare that they do not have a conflict of interest.

\section{REFERENCES}

1. Rollman BL, Brent DA. Phonotype: a New Taxonomy for mHealth Research. J Gen Intern Med. 2019.

2. Reeves B, Robinson TN, Ram N. Time for the human screenome project. Nature. 2020;577:314-317. https://doi.org/10.1038/d41586-02000032-5.

Publisher's Note: Springer Nature remains neutral with regard to jurisdictional claims in published maps and institutional affiliations.

Received November 25, 2019

Accepted March 11, 2020

Published online March 27, 2020 